\title{
O Programa Academia da Saúde - um estabelecimento de saúde da atenção básica
}

\author{
The Health Academy Program - a health establishment of Primary Care \\ El programa de la Academia de la Salud - un establecimiento de salud de Atención Primaria
}

Fabio Fortunado Brasil de Carvalho ${ }^{1}$; Patrícia Constante Jaime ${ }^{2}$

\section{Resumo}

Neste texto apresenta-se o Programa Academia da Saúde, criado em âmbito federal a partir do destaque que a atividade física ganhou nas discussões sobre a prevenção das Doenças Crônicas e do acúmulo de conhecimento gerado a partir da avaliação de experiências municipais exitosas que tinham como objetivo oferecer a prática de atividade física. Além de apresentá-lo como um estabelecimento da Atenção Básica, mediante o reconhecimento, sob a perspectiva da promoção da saúde, da importância da integração das ações de vigilância em saúde e prevenção das doenças crônicas com a Estratégia de Saúde da Família. Destaca-se a mudança ocorrida na concepção do programa, o qual surgiu relacionado principalmente à promoção da atividade física, com ela o objetivo principal passou a ser contribuir para a promoção da saúde e produção do cuidado e de modos de vida saudáveis da população a partir da implantação de polos com infraestrutura e profissionais qualificados, ampliando sua concepção. O programa incentiva a realização das atividades não apenas focadas nas doenças, sem desconsiderar ser necessário buscar respostas para o quadro epidemiológico do território, sugere-se que as atividades não busquem exclusivamente responder a polarização entre fatores de risco e fatores de proteção. Conclui-se que as estratégias que o Programa Academia da Saúde oportuniza e potencializa a construção e fortalecimento de modos de vida saudável a partir da aproximação dos polos com a vida dos usuários, do cotidiano, território, da construção de vínculo a partir do encontro, do compartilhamento de objetivos, entre outros.

Descritores: Promoção da saúde, Atenção Primária, Modos de vida saudável, Doenças Crônicas

\begin{abstract}
In this paper we present the Health Academy Program, created at the federal level from the prominence that physical activity gained in the discussions on the prevention of Chronic Diseases and generated accumulation of knowledge from the evaluation of successful local experiences that were aimed offer physical activity. In addition to present it as an establishment of Primary Care, by recognizing the perspective of health promotion, the importance of integrating surveillance actions in health and prevention of chronic diseases with the Family Health Strategy. Noteworthy is the change in the program's design, which was mainly related to the promotion of physical activity, with her main goal has to be to contribute to the promotion of health and care production and healthy lifestyles of the population from the implementation of poles with infrastructure and skilled professionals, expanding its design. The program encourages the carrying out of activities not only focused on diseases without disregarding must seek answers to the epidemiological situation of the territory, it is suggested that the activities do not seek only answer polarization between risk factors and protective factors. It is concluded that the strategies that the Health Academy Program provides opportunities and enhances the building and strengthening of healthy lifestyles from the approach of
\end{abstract}

\footnotetext{
${ }^{1}$ Escola Nacional de Saúde Pública Sérgio Arouca / Fundação Oswaldo Cruz - Ensp/Fiocruz. email: fabio.carvalho@saude.gov.br

${ }^{2}$ Faculdade de Saúde Pública da Universidade de São Paulo - FSP/USP. email: constant@usp.br
} 
ISSN 2179-6750

the poles with the lives of users, everyday, territory, construction of link from the meeting, the objectives sharing, among others.

Key-words: Health Promotion; Primary Care; Healthy lifestyles; Chronic Diseases

\section{Resumen}

En este artículo presentamos el programa de la Academia de la Salud, creada en el nivel federal de la importancia que la actividad física adquirida en los debates sobre la prevención de las enfermedades crónicas y la acumulación del conocimiento generado a partir de la evaluación de las experiencias locales exitosas que tenían como objetivo ofrecer la actividad física. Además de presentarlo como un establecimiento de la atención primaria, mediante el reconocimiento de la perspectiva de la promoción de la salud, la importancia de integrar las acciones de vigilancia de la salud y prevención de enfermedades crónicas con la Estrategia Salud de la Familia. Es de destacar el cambio en el diseño del programa, que estuvo principalmente relacionada con la promoción de la actividad física, con su principal objetivo ha de ser la de contribuir a la promoción de la salud y el cuidado de producción y estilos de vida saludables de la población de la implementación de polos con la infraestructura y los profesionales capacitados, la ampliación de su diseño. El programa fomenta la realización de actividades no sólo se centraron en las enfermedades sin dejar de lado debe buscar respuestas a la situación epidemiológica del territorio, se sugiere que las actividades no buscan única respuesta polarización entre los factores de riesgo y factores de protección. Se concluye que las estrategias que el programa de la Academia de la Salud ofrece oportunidades, además de promover la creación y el fortalecimiento de estilos de vida saludables desde el enfoque de los polos con la vida de los usuarios, todos los días, el territorio, la construcción del enlace de la reunión, compartir los objetivos, entre otros.

Palabras-claves: Promoción de la salud; Atención Primaria de Salud; Estilos de vida saludables; Enfermedades crónicas

\section{Introdução}

O estímulo à construção e fortalecimento de modos de vida saudável e da qualidade de vida para todos é um dos principais objetivos das políticas públicas de saúde na atualidade, em especial na Atenção Básica à Saúde $(\mathrm{AB})$. Através da formulação de estratégias, programas e ações, no âmbito da Saúde e de outros setores, busca-se ir além do olhar apenas para as doenças ${ }^{1,2}$.

Assim, as Políticas Públicas têm o desafio de atuar sobre as condições de saúde da população brasileira, que apresenta transições demográfica, epidemiológica e nutricional que culminam na crescente morbimortalidade por doenças e agravos não transmissíveis, considerando as influências coletivas e o contexto nos quais os sujeitos estão inseridos, além dos os hábitos de vida individuais ${ }^{2-4}$.

Neste texto apresentaremos o Programa Academia da Saúde, considerando-o uma das estratégias para alcançar a construção e fortalecimento supracitados, através da apresentação do histórico do programa, do polo - denominação da unidade na qual são desenvolvidas as atividades do programa - como um estabelecimento da $\mathrm{AB}$, a necessária articulação com a Vigilância em 
ISSN 2179-6750

Saúde (VS) e pertencente às Redes de Atenção à Saúde (RAS), desafios para a implementação e perspectivas para sua consolidação como um estabelecimento de saúde. Assim, buscar-se-á mostrar a possibilidade das atividades do Programa Academia da Saúde atuarem não só sobre os aspectos biológicos da saúde e, nesse caso, buscar aliá-los aos socioafetivos e psicológicos, em consonância com o conceito ampliado de saúde, além da valorização de outras formas de produção de saúde.

\section{Como surgiu o Programa Academia da Saúde}

A partir do lançamento da Estratégia Mundial sobre Alimentação Saudável, Atividade Física e Saúde em 2003 a atividade física ganha destaque nas discussões sobre a prevenção das Doenças Crônicas Não Transmissíveis (DCNTs) ${ }^{5}$. Em resposta ao chamamento global para construção de ações para esta agenda, o Ministério da Saúde (MS) investiu recursos financeiros, dentre outras iniciativas, para o desenvolvimento das ações prioritárias da Política Nacional de Promoção da Saúde (PNPS) publicada em 2006, dentre elas as práticas corporais e atividades físicas.

Destaca-se que existe na literatura um importante debate sobre os sentidos e significados destes termos e conceitos. Tal debate não será abordado aqui por fugir do escopo deste texto. Assim, em alguns momentos surgirão apenas um deles, busca-se respeitar a opção dos autores dos textos que foram consultados.

Malta et al ${ }^{6}$ afirmam que tais ações serviram como dispositivo indutor para o fortalecimento de ações de promoção da saúde em todas as esferas do Sistema Único de Saúde (SUS) e proporcionaram a melhoria da articulação entre diferentes áreas técnicas e de programas e políticas.

A inclusão do tema da atividade física como prioridade na PNPS passa pela compreensão de que a inatividade física é um dos principais fatores de risco para a mortalidade mundial ${ }^{7}$. Cruz e Malta ${ }^{8}$ relatam que dos municípios que receberam recursos financeiros em 2009 para as ações da PNPS, 74,8\% (total= 991 de 9 estados) informaram a implementação de programas de práticas corporais e atividades físicas. Segundo as autoras, através da experiência desses municípios, ficou evidenciada a importância do espaço físico adequado e protegido de condições climáticas, da facilidade de acesso para a participação nas atividades ofertadas, da priorização de atividades coletivas e de ser público, sem cobranças de taxas.

Houve também o reconhecimento pelo MS, sob a perspectiva da promoção da saúde, da importância da integração das ações de vigilância em saúde e prevenção de DCNTs com a Estratégia de Saúde da Família, o que foi materializado em 2008 a partir da portaria SVS n 79 de 23 de Setembro ${ }^{8}$. Assim, a AB é fortalecida na atuação sobre as DCNTs. Também em 2008 ocorre a criação do Núcleo de Apoio à Saúde da Família (Nasf). 
Em 2010 inicia-se o movimento de institucionalização das RAS, que colocaram a AB como ordenadora do cuidado, em 2011 inicia-se a implantação das primeiras redes temáticas, movimento contemporâneo à criação do programa, mas não é possível afirmar em um primeiro momento que há relação entre o programa e as redes. A primeira aproximação mais robusta ocorre em 2013 com a Rede de Atenção à Saúde das Pessoas com Doenças Crônicas e revisão das portarias do programa. A relação do Programa Academia da Saúde com as RAS será explorada posteriormente no texto.

Já em 2011, com fortalecimento dos Nasf como uma das principais estratégias da Política Nacional de Atenção Básica (PNAB) avança-se, nas Secretarias de Atenção à Saúde (SAS) e Secretaria de Vigilância em Saúde (SVS) do MS, na construção de um programa nacional, no âmbito do SUS, de promoção da atividade física.

Assim, o Programa Academia da Saúde é criado em 2011 baseado no acúmulo de conhecimento gerado a partir, dentre outras, das ações supracitadas e da avaliação dos Programas Academia da Cidade em Recife - PE, Aracaju - SE e Belo Horizonte - MG; o Programa CuritibAtiva em Curitiba - PR, o Serviço de Orientação ao Exercício de Vitória - ES ${ }^{9}$, experiências municipais exitosas que tinham como objetivo oferecer a prática de atividade física para a comunidade. Em comum entre estes programas havia a ampla participação da comunidade e a existência de espaços públicos especialmente construídos para o desenvolvimento das ações dos programas, corroborando o achado relatado por Cruz e Malta ${ }^{8}$.

Destaca-se ainda a inclusão de indicadores e metas relacionados à implementação do Programa Academia da Saúde, em 2011, no Plano Nacional de Saúde (2011-2015), no Planejamento Estratégico do MS (2011-2015) e no Contrato Organizativo de Ação Pública (COAP) ${ }^{6}$. O COAP é o acordo de colaboração firmado entre entes federativos com a finalidade de organizar e integrar as ações e serviços de saúde na rede regionalizada e hierarquizada, com definição de responsabilidades, indicadores e metas de saúde, critérios de avaliação de desempenho, recursos financeiros que serão disponibilizados, forma de controle e fiscalização de sua execução e demais elementos necessários à implementação integrada das ações e serviços de saúde ${ }^{10}$.

\section{O Programa Academia da Saúde}

O programa é incentivado pelo MS por meio de recursos financeiros para a construção dos polos em três modalidades: básica, intermediária e ampliada, além de recursos financeiros para o custeio das atividades. O primeiro tipo de recurso é pago, em repasse do Fundo Nacional de Saúde (FNS) para o Fundo Municipal de Saúde (FMS), conhecido como: fundo a fundo, em três parcelas, de acordo com o estágio de construção dos polos, já o segundo, varia de acordo com a existência ou 
ISSN 2179-6750

não do Nasf no município. Este recurso também é ofertado para os programas e iniciativas similares ao Programa Academia da Saúde, que são programas municipais que já existem e são reconhecidos pelo MS como tal, tornando-se aptos a fazer a solicitação do recurso de custeio. Os estados e municípios também podem realizar o aporte de recursos financeiros para as duas situações descritas, conforme pactuação.

O polo do Programa Academia da Saúde é constituído por três ambientes: Espaço de Vivência, Espaço Multiuso e Espaço com equipamentos com a Barra Tripla Horizontal, Barras paralelas, espaldar, bancos, pranchas para exercícios abdominais e barras marinheiro. As diferentes modalidades possuem metragens diferentes, além de, nas modalidades intermediária e ampliada, o Espaço de Vivência contar com uma estrutura de apoio. Para detalhamento das estruturas dos polos do Programa Academia da Saúde, sugerimos a leitura do Programa de Necessidades da estrutura física mínima constante no anexo I da Portaria GM/MS n 2.684, de 8 de Novembro de 2013 (republicada no dia 14 de Novembro de 2013) ${ }^{11}$ ou de outras normativas que tratarem do tema.

Todos os ambientes dos polos foram pensados para propiciar a possibilidade de utilização no desenvolvimento das atividades. Merece especial atenção no planejamento das atividades o espaço de equipamentos, já que há necessidade de certo grau de força e resistência muscular por parte dos usuários dos polos para realizar as atividades nas barras triplas horizontais e nas paralelas. Já os demais equipamentos possuem a recomendação, através de croquis, disponíveis em: www.saude.gov.br/academiadasaude, da construção em diferentes alturas e angulações para que tornem possível a progressão em sua utilização, ou seja, uns são mais fáceis, permitindo que uma gama maior de participantes possa utilizá-los.

O espaço de vivência, construção coberta, é a resposta à evidência que mostrou a importância do espaço físico protegido de condições climáticas, e o espaço multiuso possibilita o desenvolvimento de atividades diversas, que contemplam todos os eixos de atividades do Programa.

Apresentados, de forma geral, os recursos e a estrutura dos polos, é importante destacar a mudança ocorrida na concepção do Programa Academia da Saúde em 2013. Conforme abordado no histórico, o programa surgiu relacionado principalmente à promoção da atividade física. Com a mudança, o objetivo principal do programa passou a ser: contribuir para a promoção da saúde e produção do cuidado e de modos de vida saudáveis da população a partir da implantação de polos com infraestrutura e profissionais qualificados ${ }^{11}$. Entre os objetivos específicos, destacam-se:

- Ampliar o acesso da população às políticas públicas de promoção da saúde;

- Desenvolver a atenção à saúde nas linhas de cuidado;

- Promover a convergência de projetos ou programas nos âmbitos da saúde, educação, cultura, 
ISSN 2179-6750

assistência social, esporte e lazer;

- Ampliar a autonomia dos indivíduos sobre as escolhas de modos de vida mais saudáveis;

- Aumentar o nível de atividade física da população;

- Contribuir para ampliação e valorização da utilização dos espaços públicos de lazer.

Tal reformulação reforça a concepção do Programa Academia da Saúde na qual ele deve ser planejado e operacionalizado na lógica da $\mathrm{AB}$, com destaque para a compreensão da saúde como direito e produção social, da proporção entre a ação coletiva e individual, do trabalho transdisciplinar em equipe multiprofissional e do estímulo à participação social.

Através da publicação da nova portaria - Portaria GM/MS n 2.681, de 7 de Novembro de $2013^{12}$ - na qual as práticas corporais e atividades físicas são um dos eixos de atividades a serem desenvolvidos nos polos, o programa que surgiu a partir do destaque dado à atividade física como fator de proteção contra as DCNTs e de experiências municipais nas quais esta era a principal atividade ofertada é possível afirmar que ele passa a ter uma concepção ampliada, como um programa que valoriza outras formas de produção de saúde para além dos benefícios biológicos da atividade física, sem desconsiderar a importância desta.

Os outros eixos de atividades são: Promoção da alimentação saudável; Produção do Cuidado e modos de vida saudável; Mobilização da comunidade; Educação em Saúde; Práticas Integrativas e Complementares; Práticas artísticas e culturais; Planejamento e gestão. Destaca-se que não há uma hierarquia entre eles, todos são importantes e é desejável que todos sejam desenvolvidos nas atividades. Ressalta-se a grande possibilidade de articulação dessas atividades, por exemplo, tendo como tema uma festa junina ou outra festa regional é possível desenvolver atividades que englobem a maioria ou todos os eixos de atividades.

É possível afirmar que a mudança citada na concepção do programa ainda é um grande desafio já que o histórico citado criou a compreensão de que o Programa Academia da Saúde se restringe à oferta de atividade física, muitas vezes reproduzindo, exclusivamente, uma grade de atividades semelhante ao das academias de ginástica comerciais. Destaca-se que as práticas corporais e atividades físicas são ferramentas importantes para a saúde individual e coletiva ${ }^{13-16}$, mas não são a única possibilidade de produção de saúde no Programa Academia da Saúde. Costa et al ${ }^{1}$ afirmam que locais de promoção da saúde, como as Academias da Cidade, no caso de Belo Horizonte - MG, devem incorporar medidas que não se direcionam a uma determinada enfermidade ou desordem, mas objetivam propiciar saúde e bem estar.

Outra compreensão ainda vigente é a separação da AB e da VS na gestão do programa, a expertise que a VS adquiriu nos programas de promoção da atividade física e o histórico desafio de 
ISSN 2179-6750

articulação entre estas áreas acabou por criar barreiras para a gestão do Programa Academia da Saúde, que necessariamente deve ser articulado entre as duas áreas.

Assim, fica evidenciado que essas duas questões citadas ainda são um grande desafio para a implementação do Programa Academia da Saúde. Além dessas, apresentaremos outros desafios em tópico posterior do texto.

\section{Um estabelecimento de saúde da Atenção Básica}

A partir de 2011 o MS realizou uma série de medidas que fortaleceram o papel e a importância da $\mathrm{AB}$ no conjunto de políticas da gestão federal do SUS, dentre elas o aumento do financiamento federal e o investimento na ampliação e qualificação da infraestrutura das Unidades Básicas de Saúde (UBS) ${ }^{17}$. O Programa Academia da Saúde também pode ser considerado como uma dessas medidas.

$\mathrm{A} \mathrm{AB}$ é caracterizada por um "conjunto de ações de saúde, no âmbito individual e coletivo, que abrange a promoção e a proteção da saúde, a prevenção de agravos, o diagnóstico, o tratamento, a reabilitação, a redução de danos e a manutenção da saúde" ${ }^{2}$. Para impactar na situação de saúde e na autonomia de sujeitos e coletividades e nos determinantes e condicionantes da saúde, o objetivo da $\mathrm{AB}$ é desenvolver uma atenção integral, assumindo responsabilidade sanitária sobre uma população e um território definidos, levando em conta a dinamicidade desse território e utilizando o trabalho em equipe ${ }^{2}$.

A partir das definições apresentadas pela PNAB, afirma-se que o polo do Programa Academia da Saúde compõe a rede de serviços de saúde da AB. Destaca-se a aproximação dos serviços de saúde da $\mathrm{AB}$ com o território e com a vida dos usuários, por considerar a dinamicidade do território e a singularidade e inserção sociocultural dos sujeitos, potencializando o cuidado integral, entendido como os arranjos planejados e operacionalizados pelos serviços e equipes de saúde da $\mathrm{AB}$ a fím de que os usuários tenham acesso a respostas para suas necessidades de saúde ${ }^{18}$. Nesse texto, as referências ao princípio da integralidade e aos termos: cuidado integral; integralidade da atenção à saúde; serão considerados com sentidos semelhantes ao apresentado nesse momento.

As atividades do Programa Academia da Saúde, como um estabelecimento de saúde da AB, aumentam a potencialidade relacionada à construção de vínculo com os usuários por propiciar o encontro, a criação e o compartilhamento de objetivos coletivos, a ludicidade, entre outros, o que muitas vezes pode não ser possível na prática clínica. Com isso, pode aumentar a resolutividade das ações de saúde, o que para Silva e Carvalho ${ }^{19}$ abrange a conquista de melhores níveis de saúde, a 
ISSN 2179-6750

mudança do perfil epidemiológico e a garantia de bem-estar da comunidade à qual presta atenção à saúde de forma permanente e não pontual, não se restringindo ao processo de curar e de 'dar alta a um paciente'.

O acesso universal e contínuo no polo do Programa Academia da Saúde é representado pela priorização do trabalho em grupos, o que não impede da abordagem individual ser utilizada. Não iremos explorar a questão do acesso universal e contínuo por não ser objeto deste texto, mas destaca-se que o não se encerra nesta priorização. É importante analisar as possibilidades que aumentem o número de beneficiários das ações e atividades através do planejamento participativo que contemple as potencialidades e desejos da comunidade na qual o polo está inserido. Faz-se necessário estar atento para a criação de barreiras de acesso como a obrigatoriedade de apresentação de atestados médicos, independente da situação de saúde dos sujeitos, ou roupas ditas 'adequadas' para a participação nas atividades, entre outras.

O programa incentiva a realização das atividades não apenas focadas nas doenças, sem desconsiderar ser necessário buscar respostas para o quadro epidemiológico do território. As DCNTs são um exemplo, sugere-se que as atividades não busquem exclusivamente responder a polarização entre fatores de risco e fatores de proteção para elas, também se sugere não haver a divisão de usuários exclusivamente por condições de saúde ou ciclo de vida, grupo de idosos ou grupo de hipertensos são exemplos.

Caso sejam feitas tais opções, é essencial que existam momentos (regulares) nos quais haja a interação entre os usuários, é importante destacar aqui que a intergeracionalidade, entendida como a aproximação e a convivência entre diferentes gerações, é um dos princípios do programa.

Além disso, até mesmo os benefícios biológicos das atividades do Programa Academia da Saúde para a prevenção das DCNTs, em especial os proporcionados pela alimentação saudável e pelas práticas corporais e atividades físicas, carecem de abordagem na qual os princípios e diretrizes da $\mathrm{AB}$ estejam presentes, ou seja, buscar-se-á apoiar os sujeitos para que possam agir em favor de sua saúde através dessas atividades levando em conta sua história de vida, sua relação com a própria saúde, desejos, objetivos, seu saber e não apenas marcadores biológicos na normalidade.

O que foi abordado até aqui mostra a importância da corresponsabilização na construção de projetos terapêuticos, o que pressupõe o diálogo entre o saber baseado nas experiências de vida dos sujeitos e o dos trabalhadores da saúde, baseados em sua formação técnico-científica. Tal diálogo e o acompanhamento da evolução de situação de saúde ao longo do tempo (longitudinalidade do cuidado) ampliam a autonomia de ambos na construção dos projetos terapêuticos e favorece a participação dos usuários, a partir do empoderamento e aumento da autoestima dos sujeitos e 
ISSN 2179-6750

coletividades, fortalecendo o cuidado consigo e a relação do sujeito com seu território.

A multiprofissionalidade também é essencial para o programa, do ponto de vista normativo há um rol de profissionais, entre eles o arteterapeuta, sanitarista, nutricionista, profissional de educação física na saúde, ente outros, que devem ser cadastrados no Cadastro Nacional de Estabelecimentos de Saúde (CNES) para que o ente federativo se torne apto a receber o incentivo de custeio, mas é desejável que diversos trabalhadores da $\mathrm{AB}$ desenvolvam atividades nos polos, tais como os profissionais da enfermagem e da medicina, integrantes da equipe mínima da $\mathrm{AB}$, que não fazem parte desse rol.

Em especial as equipes do Nasf por terem como uma das características do seu processo de trabalho o apoio matricial, o que permitirá uma ampliação do escopo de atividades ofertadas pelo programa, bem como um aprofundamento nas questões relativas à continuidade do cuidado por ser, muitas vezes, um elo entre os profissionais do polo e das unidades de saúde do território. A atuação desses profissionais deve ser articulada e integrada com os demais estabelecimentos da $\mathrm{AB}$ e da rede de saúde.

Ainda em relação ao acesso ao Programa não há necessidade de consideração rígida de um território de abrangência, ou seja, todos podem participar independente da localidade na qual residam. Ter um território de referência auxilia para organizar as ações de planejamento e monitoramento tendo como base a realidade local, seus problemas e suas potencialidades. Porém podem existir questões práticas que impeçam que toda a população participe de determinada atividade, como o espaço físico disponível, caso isso ocorra o planejamento deverá ser refeito para tentar atender a essa demanda.

As características do programa apresentadas até aqui possibilitam que ele oportunize acesso à $\mathrm{AB}$ a sujeitos e coletividades que historicamente apresentam dificuldades, por exemplo, aos adolescentes e aos homens. Destacam-se aqui as práticas artísticas e culturais como o grafite e o Hip-Hop para os jovens, as práticas corporais e atividades físicas, com seu amplo leque de possibilidades de modalidades e variações para os homens, ou ainda a educação em saúde para ambos.

Ferramentas operacionais para o processo de trabalho na $\mathrm{AB}$ como o apoio matricial entre os serviços, a regulação através da estratificação de risco, projetos terapêuticos singulares, protocolos de atenção, entre outros, também são importantes para o processo de trabalho dos profissionais do Programa Academia da Saúde.

Baseado nas características da $\mathrm{AB}$ exploradas até aqui, afirma-se que o polo do Programa Academia da Saúde, na sua articulação com as demais unidades de saúde da AB, é uma possível 
ISSN 2179-6750

porta de entrada das RAS, fortalece a rede de serviços e ações de saúde da $A B$, em especial ao ampliar o escopo de ações, contribui na coordenação do cuidado, favorece a integralidade e resolutividade a partir da integração de ações programáticas e da demanda espontânea, e contribui na ordenação das redes. Nos municípios onde isso não ocorre, faz-se necessário construir essa possibilidade.

\section{O Programa Academia da Saúde nas Redes: de Atenção à Saúde e de Produção Social da}

\section{Saúde}

Neste momento buscaremos avançar na compreensão do polo do Programa Academia da Saúde como um ponto de atenção das RAS e como um estabelecimento de saúde da AB nas redes de produção social da saúde através da intersetorialidade. Configurar-se como ponto de atenção da RAS, tornando-se complementar e potencializador das ações de cuidados individuais e coletivos na $\mathrm{AB}$, além de um espaço de produção, ressignificação e vivência de conhecimentos favoráveis à construção coletiva de modos de vida saudáveis são diretrizes do Programa Academia da Saúde ${ }^{12}$.

As RAS são os arranjos organizativos formados por ações e serviços de saúde com diferentes configurações e densidades tecnológicas, que integrados por meio de sistemas de apoio técnico, logístico e de gestão, buscam garantir a integralidade do cuidado ${ }^{20}$. A implantação das RAS é a principal agenda assistencial no SUS, em conjunto com a $\mathrm{AB}^{21}$, trata-se de uma iniciativa com vistas à maior integração, regionalmente, entre os serviços de saúde, com o objetivo de garantir a integralidade da atenção à saúde ${ }^{22}$.

Para Magalhães Júnior ${ }^{21}$ a hegemônica fragmentação do SUS impede a efetivação dos princípios da universalidade, equidade e integralidade. Assim as RAS são uma estratégia para superar esta fragmentação da atenção e da gestão à saúde para aperfeiçoar o funcionamento do SUS, visando assegurar o cuidado integral ao usuário ${ }^{23}$. Para Costa e Santos ${ }^{24}$ o princípio da integralidade adquire concretude por meio das RAS através de conexões e integração das ações e dos serviços de saúde nos territórios. Com isso, o alcance do objetivo do SUS relacionado à efetivação da integralidade é fortalecido através da superação do modelo fragmentado de atenção à saúde.

Destacam-se entre as principais características das RAS: a AB como ordenadora e centro de comunicação, sendo o contato preferencial de sujeitos e coletividades com os serviços de saúde ${ }^{19}$, a formação de relações horizontais entre os pontos de atenção à saúde, a centralidade nas necessidades de saúde da população, a responsabilização por atenção contínua e integral, o cuidado multiprofissional e o compartilhamento de objetivos e o compromisso com resultados sanitários e 
econômicos ${ }^{23}$.

As RAS significam a transformação do funcionamento dos serviços e do processo de trabalho das equipes, exigindo de seus atores maior capacidade de análise, intervenção e autonomia para a construção de práticas transformadoras ${ }^{20}$.

Dentro das RAS, foram constituídas cinco Redes Temáticas, a saber:

1. Rede Cegonha.

2. Rede de Atenção às Urgências e Emergências (RUE).

3. Rede de Atenção Psicossocial (RAPS).

4. Rede de Cuidado à Pessoa com Deficiência.

5. Rede de Atenção à Saúde das Pessoas com Doenças Crônicas (RASPDC).

É importante destacar que os pontos de atenção à saúde se diferenciam pela densidade tecnológica, mas não há uma hierarquia, ou seja, um não é mais importante que o outro. Além dos pontos de Atenção à Saúde, são necessários sistemas de apoio: serviços de diagnóstico terapêutico, assistência farmacêutica e sistemas de informação em saúde ${ }^{23}$.

As atividades do Programa Academia da Saúde podem ser relacionadas à produção de cuidado e promoção da saúde de usuários do SUS das redes existentes. Destacam-se as características do Programa na $\mathrm{AB}$ e a partir daí é necessário planejar e operacionalizar as ações articuladas nas RAS. Chueiri et al. ${ }^{25}$ afirmam que o Programa Academia da Saúde é uma estratégia complementar à RASPDC, com foco no cuidado integral e a promoção da saúde. Há também a inclusão do programa nas diretrizes para a organização da prevenção e do tratamento do sobrepeso e obesidade como linha de cuidado prioritária RASPDC ${ }^{26}$. Jorge et al ${ }^{22}$ afirmam que o programa pode contribuir para a RUE através da prevenção, o tratamento e a reabilitação de doenças cárdio e cerebrovasculares.

Assim, as situações, agudas ou crônicas, nas quais os sujeitos necessitam utilizar os serviços de saúde devem ser avaliadas. Nas situações agudas deve haver o atendimento em qualquer ponto de atenção de acordo com a gravidade e complexidade do caso. Já nas crônicas, o atendimento deve ocorrer preferencialmente na AB. Em ambos os casos, o Programa Academia da Saúde e os demais serviços da $\mathrm{AB}$ deverão ser responsáveis pela busca da longitudinalidade do cuidado à saúde utilizando tecnologias de gestão do cuidado que ampliem a autonomia e empoderamento de sujeitos e coletividades ${ }^{20}$.

Dentre os desafios para a implementação das RAS destacam-se: criação de cultura e práticas de trabalho em rede, planejamento regional (planos de ação regionais), especificidades loco regionais, formação, qualificação e Educação Permanente (EP) dos trabalhadores. Além destes, 
ISSN 2179-6750

Magalhães Júnior e Pinto ${ }^{17}$ relatam que a $\mathrm{AB}$, de modo geral, ainda não está organizada nem empoderada para realizar a coordenação do cuidado e ordenação do acesso nas RAS. Contudo, estes autores afirmam que existem iniciativas abordando as questões que impedem que a $\mathrm{AB}$ funcione plenamente em relação às RAS.

Assim, os pontos de atenção à saúde nas RAS devem operar a partir da compreensão dos determinantes e condicionantes no processo de produção da saúde, da necessária articulação com os demais pontos de atenção à saúde e dos estabelecimentos das redes de produção social da saúde, do trabalho transdisciplinar em equipe multiprofissional, do estímulo à participação social, entre outros 19 .

Já a rede de produção social da saúde refere-se à intersetorialidade nas políticas, programas e ações de saúde. A intersetorialidade na construção e desenvolvimento das ações é um dos princípios do Programa Academia da Saúde ${ }^{12}$, segundo Carvalho ${ }^{27}$ a intersetorialidade busca superar a especialização e setorialização com o objetivo de integrar saberes e percepções dos sujeitos e de sua realidade social considerando-os em sua integralidade tendo como principal objetivo a promoção de mudanças nas condições de vida e de saúde da população mediante a visão integrada dos complexos problemas sociais da atualidade, o que requer a inclusão de vários atores sociais e seus olhares, além da interação das diversas políticas sociais através de medidas multidisciplinares.

Mais do que abordar as características dessas redes: de atenção à saúde e de produção social da saúde, importa apontar para a necessária articulação entre elas. Retomamos aqui os objetivos específicos do programa já citados, os quais se relacionam ao acesso da população às políticas públicas de promoção da saúde, à convergência de projetos ou programas nos âmbitos da educação, cultura, assistência social, esporte e lazer; e à valorização da utilização dos espaços públicos de lazer para ressaltar a abertura e necessidade que o Programa Academia da Saúde possui para a articulação intersetorial.

Iniciativas de ações intersetoriais no Programa Academia da Saúde são representadas, entre outras, pela busca de articulação em meados de 2014 com o Programa Esporte e Lazer da Cidade (PELC) - proporciona a prática de atividades físicas, culturais e de lazer que envolva todas as faixas etárias, incluindo as com deficiência, estimula a convivência social, a formação de gestores e lideranças comunitárias, fomenta a pesquisa e a socialização do conhecimento, contribuindo para que o esporte e o lazer sejam tratados como políticas públicas e direito de todos - do Ministério do Esporte, com o Plano Juventude Viva - criado pelo governo federal fruto de uma intensa articulação interministerial para enfrentar a violência contra a juventude brasileira, especialmente os jovens 
ISSN 2179-6750

negros - da Secretaria Nacional de Juventude da Presidência da República ${ }^{28}$.

\section{Desafios na implementação do Programa Academia da Saúde}

Atualmente existem mais de 3.700 polos habilitados, mais de 1.500 concluídos em aproximadamente 2.850 municípios brasileiros, dos quais 856 informaram estar em funcionamento 29,30. É possível afirmar que recentemente a implementação do Programa Academia da Saúde apresenta uma transição: o foco exclusivo era a construção dos polos, agora, apesar da permanência desta questão, segundo dados do MS há um crescimento importante no número de polos concluídos, levando os atores envolvidos na gestão federal, estadual e municipal do programa a construir e debater possibilidades de planejamento, organização e oferta de atividades que permitam o alcance dos objetivos já citados.

Em um primeiro momento, iniciado a partir do primeiro semestre de 2012 quando os municípios com polos habilitados receberam a primeira parcela do recurso de construção dos polos, é possível afirmar que esses municípios não tinham a clareza das potencialidades do Programa Academia da Saúde, devido em grande parte à sua recente criação.

Algumas hipóteses sobre essa falta de clareza relacionam-se: ao não envolvimento, por parte dos municípios, de trabalhadores da saúde da $\mathrm{AB}$ na confecção dos projetos, levando a construções que não permitiam a efetiva utilização dos ambientes ou equipamentos. Aliado a isso, ocorreu um forte apelo comercial para os equipamentos das Academias da Terceira Idade - ATIs, ou Academias ao Ar Livre, tais como aqueles que simulam os que são encontrados nas academias comerciais: Pressão de Pernas (Leg Press), Elípticos (Transport), Puxadores, entre outros. Foge ao escopo deste artigo analisar esses equipamentos, o fato é que eles não fazem parte do programa de necessidades da estrutura física mínima dos polos do Programa Academia da Saúde.

Outra questão que possivelmente influenciou este momento foi a mudança da gestão municipal em janeiro de 2013. O acúmulo de informações e debates sobre o Programa Academia da Saúde e suas potencialidades, muitas vezes, se perdeu, fazendo o ciclo ser novamente iniciado.

O segundo momento da implementação fica evidenciada a partir de fevereiro de 2014 quando algumas experiências exitosas foram apresentadas no II Seminário Nacional e na I Mostra de Práticas do Programa Academia da Saúde, realizados em Brasília (DF), destacam-se as que estão ocorrendo em Mâncio Lima (AC), Rio Claro (RJ), Recife (PE), que conseguiram envolver grande parte da população em ações de promoção da saúde e produção do cuidado. Assim, os atores envolvidos na gestão e na operacionalização do Programa Academia da Saúde discutem e constroem possibilidades para que o programa possa atingir seus objetivos, contribuindo assim para 
ISSN 2179-6750

o cuidado integral, na $\mathrm{AB}$, de indivíduos e coletividades.

Sá et al ${ }^{29}$ afirmam que nos polos em funcionamento são desenvolvidos prioritariamente atividades de práticas corporais e atividades físicas, alimentação saudável e educação em saúde. O principal público participante do Programa foram adultos e idosos, a inclusão de crianças e adolescentes foi apontada como umas das principais dificuldades. As autoras defendem o potencial do Programa como estratégia de promoção da saúde e produção do cuidado nas comunidades, sendo fundamental qualificar suas ações em todo o país. Carvalho e Nogueira ${ }^{31}$ apresentam dados da literatura que corroboram com essas afirmativas.

Destacam-se os principais desafios: a superação da concepção hegemônica de saúde que privilegia os benefícios biológicos, em especial das práticas corporais e atividades físicas; e da promoção da alimentação saudável. É essencial atuar sobre o atual quadro epidemiológico brasileiro que tem as DCNTs como uma das principais causas de morbimortalidade, contudo, é necessário aliar os benefícios biológicos aos socioafetivos e psicológicos, levando em consideração os determinantes e condicionantes da saúde e, assim, valorizando outras formas de produção de saúde.

\section{Considerações}

Concluímos afirmando que as estratégias que o Programa Academia da Saúde, como um estabelecimento de saúde da $\mathrm{AB}$, oportuniza e potencializa para alcançar a construção e fortalecimento de modos de vida saudável e da qualidade de vida são relacionadas: à aproximação dos polos com a vida dos usuários, do cotidiano e da realidade pulsante do território; à construção de vínculo com os usuários a partir do encontro, do compartilhamento de objetivos; ao acesso universal e contínuo, com a superação de barreiras de acesso; à corresponsabilização na construção de projetos terapêuticos e com isso a ampliação da autonomia de usuários e trabalhadores da saúde, a partir do empoderamento e aumento da autoestima dos sujeitos e coletividades, fortalecendo o cuidado integral e permitindo o aumento da resolutividade das ações de saúde.

Os desafios evidenciados mostram que ainda há um longo caminho a ser percorrido pelos atores sociais e institucionais envolvidos na implementação e operacionalização do Programa Academia da Saúde. A construção e fortalecimento de modos de vida saudável e da qualidade de vida para indivíduos e coletividades é complexa e processual, assim como a promoção da saúde e produção do cuidado objetivadas pelas atividades do programa.

Sobre estes desafios, são reconhecidos e vislumbrados pelo Programa Academia da Saúde como ponto de partida para a busca por melhores condições de vida e de saúde de seus usuários no cotidiano dos processos de trabalho e nos territórios, enfim, nos polos e nos demais serviços de 
ISSN 2179-6750

saúde da AB. Assim, também é importante destacar que já estão ocorrendo diversas experiências nos polos do programa que mostram impactos positivos na vida dos usuários da $\mathrm{AB}$.

\section{Agradecimentos}

Esse capítulo é dedicado à equipe do Programa Academia da Saúde e demais integrantes do Departamento de Atenção Básica do Ministério da Saúde - DAB/SAS/MS.

À Dra. Giannina do Espírito Santo Wildhagen já que esse texto foi produzido inicialmente para um projeto editorial sob sua coordenação e foi gentilmente cedido para a publicação nesse periódico.

\section{Referências}

1. Costa BVL, Mendonça RD, Santos LC, Peixoto SV, Alves M, Lopes ACS. Academia da Cidade: um serviço de promoção da saúde na rede assistencial do Sistema Único de Saúde. Ciência \& Saúde Coletiva, 18(1):95-102, 2013.

2. Brasil. Ministério da Saúde. Secretaria de Atenção à Saúde. Departamento de Atenção Básica. Política Nacional de Atenção Básica - Brasília: Ministério da Saúde, 2012.

3. . Ministério da Saúde. Instituto Brasileiro de Geografia e Estatística. Ministério do Planejamento, Orçamento e Gestão. Pesquisa nacional de saúde: percepção do estado de saúde, estilos de vida e doenças crônicas. Brasil, grandes regiões e unidades da federação. Rio de Janeiro, 2014.

4. Chueiri PS, Harzheim E, Gauche H, Vasconcelos LLC. Pessoas com doenças crônicas, as redes de atenção e a Atenção Primária à Saúde. Divulgação em Saúde para debate | Rio de Janeiro , n. 52, p. 114-124, Out 2014.

5. Cruz DKA. Da promoção à prevenção: o processo de formulação da política nacional de promoção da saúde no período de 2003 a 2006. [Dissertação]. Recife (PE) 2010.

6. Malta DC, Silva MMA, Albuquerque GM, Lima CM, Cavalcante T, Jaime PC et al. A implementação das prioridades da Política Nacional de Promoção da Saúde, um balanço, 2006 a 2014. Ciência \& Saúde Coletiva, 19(11):4301-4311, 2014.

7. Malta DC, Silva MMA, Albuquerque GM, Amorim RCA, Rodrigues GBA, Silva TS et al. Política Nacional de Promoção da Saúde, descrição da implementação do eixo atividade física e práticas corporais, 2006 a 2014. Rev Bras Ativ Fis e Saúde. Pelotas/RS. 19(3):286299. 2014.

8. Cruz DKA, Malta DC. Práticas corporais e atividade física no Sistema Único de Saúde: das experiências locais à implementação de um programa nacional. In: Benedetti TRB et al. 
ISSN 2179-6750

Orgs. A formação do profissional de educação física para o setor saúde. Florianópolis. Postmix, 2014.

9. Brasil. Ministério da Saúde. Secretaria de Vigilância em Saúde. Avaliação de Efetividade de Programas de Atividade Física no Brasil. Brasília: Ministério da Saúde, 2011.

10. Brasil. Decreto $n^{\circ} 7508$ de 28 de junho de 2011. Regulamenta a Lei ${ }^{\circ} 8.080$, de 19 de setembro de 1990, para dispor sobre a organização do Sistema Único de Saúde - SUS, o planejamento da saúde, a assistência à saúde e a articulação interfederativa, e dá outras providências. 2011.

11. Ministério da Saúde. Portaria GM/MS n ${ }^{\circ} 2684$ de 8 de Novembro de 2013. Redefine as regras e os critérios referentes aos incentivos financeiros de investimento para construção de polos e de custeio e no âmbito do Programa Academia da Saúde e os critérios de similaridade entre Programas em Desenvolvimento no Distrito Federal ou no Município e o Programa Academia da Saúde, 2013.

12. ___ Ministério da Saúde. Portaria GM/MS nº 2681 de 7 de Novembro de 2013. Redefine o Programa Academia da Saúde no âmbito do Sistema Único de Saúde - SUS, 2013.

13. Bagrichevsky M, Estevão A. Perspectivas para a formação profissional em educação física: o SUS como horizonte de atuação. Arquivos em Movimento. 2008. v.4 Nº 1. p.128-143.

14. Andrade DR. Políticas recentes de promoção da atividade física no Brasil. Revista Corpoconsciência. 2011. v. 15, n. 2, p. 02-06.

15. Malta DC, Castro AM, Cruz DKA, Gosh CS. A promoção da saúde e da atividade física no Sistema único de Saúde. Revista Brasileira de Atividade Física \& Saude. 2008. v.13, Numero 1. p. 24-27.

16. Carvalho FFB. Análise crítica da carta brasileira de prevenção integrada na área da saúde na perspectiva da Educação Física através do enfoque radical de promoção da saúde. Saúde e Sociedade. 2009. v.18, n. 1, p. 227-236

17. Magalhães Júnior HM, Pinto HA. Atenção Básica enquanto ordenadora da rede e coordenadora do cuidado: ainda uma utopia? Revista Divulgação em Saúde para debate. Rio de Janeiro, $\mathrm{n}^{\circ}$ 51. p.14-29, 2014.

18. Tura LMT, Soares K, Casartelli CH. Atenção Primária em Saúde. In: Souza MF, Franco MS, Mendonça AVM, Organizadores. Saúde da família nos municípios brasileiros: os reflexos dos 20 anos do espelho do futuro. Campinas, SP. Saberes Editora. 2014. p.178-205. 
ISSN 2179-6750

19. Silva TS, Carvalho FFB. A Promoção da Saúde na Atenção Primária: Possibilidades e desafios. In: Programa de Atualização em Enfermagem Atenção Primária e Saúde da Família. Artmed Panamericana Editora. Porto Alegre/RS, 2014.

20. Brasil. Portaria n. 4.279, de 30 de dezembro de 2010. Estabelece diretrizes para a organização da Rede de Atenção à Saúde no âmbito do Sistema Único de Saúde. Brasília, 2010 .

21. Magalhães Júnior HM. Redes de Atenção à Saúde: rumo à integralidade. Revista Divulgação em Saúde para debate. Rio de Janeiro, n 52. p.15-37, 2014.

22. Jorge AO, Coutinho AAP, Cavalcante APS, Fagundes MAS, Pequeno CC, Carmo M et al. Entendendo os desafios para a implementação da Rede de Atenção às Urgências e Emergências no Brasil: uma análise crítica. Revista Divulgação em Saúde para debate. Rio de Janeiro, $\mathrm{n}^{\circ}$ 52, p. 125-145, Out 2014

23. Brasil. Ministério da Saúde. Secretaria de Atenção à Saúde. Implantação das Redes de Atenção à Saúde e outras estratégias da SAS / Ministério da Saúde. Secretaria de Atenção à Saúde. - Brasília: Ministério da Saúde, 2014.

24. Costa AM, Santos FP. Editorial: O processo de construção das Redes de Atenção à Saúde. Revista Divulgação em Saúde para debate. Redes de Atenção à Saúde: construindo o cuidado integral. Rio de Janeiro, $\mathrm{n}^{\circ}$ 52. p.4-7, 2014.

25. Chueiri PS, Harzheim E, Gauche H, Vasconcelos LLC. Pessoas com doenças crônicas, as redes de atenção e a Atenção Primária à Saúde. Divulgação em Saúde para Debate. Rio de Janeiro , n. 52, p. 114-124, Out 2014.

26. Brasil. Portaria GM/MS No 424, de 19 de março de 2013. Redefine as diretrizes para a organização da prevenção e do tratamento do sobrepeso e obesidade como linha de cuidado prioritária da Rede de Atenção à Saúde das Pessoas com Doenças Crônicas.

27. Carvalho FFB. A saúde na escola e a Promoção da Saúde: conhecendo a dinâmica intersetorial em Duque de Caxias e Nova Iguaçu - RJ. Rio de Janeiro - RJ. Dissertação (Mestrado em Saúde Pública) - Escola Nacional de Saúde Pública, Fundação Oswaldo Cruz, 2012.

28. Morais B. Maracanaú inicia hoje capacitação do projeto Piloto da Academia da Saúde. Disponível em http://www.maracanau.ce.gov.br/noticia-saude/item/13724-maracanauinicia-hoje-capacitacao-do-projeto-piloto-da-academia-da-saude. 2014. Acesso em 05 de janeiro de 2015. 
ISSN 2179-6750

29. Sá GBAR, Dornelles GC, Cruz KG, Amorim RCA, Andrade SSCA, Oliveira TP et al. O Programa Academia da Saúde como estratégia de promoção da saúde e modos de vida saudáveis: cenário nacional de implementação. Ciência \& Saúde Coletiva, 21(6):1849-1859, 2016.

30. Brasil. Ministério da Saúde. Dados da gestão do Programa Academia da Saúde. Disponível em: www.saude.gov.br/academiadasaude. Acesso em 28 de novembro de 2014.

31. Carvalho FFB, Nogueira JAD. Práticas corporais e atividades físicas na perspectiva da Promoção da Saúde na Atenção Básica. Ciência \& Saúde Coletiva, 21(6):1829-1838, 2016. 